\title{
Lifting Technology Research and Application of the Oversize Gear Ring
} Shiyin Liu, Xiaoliang Du

School of Mechanics \& Electrics, Xi'an University of Architecture and Technology, Xi'an 710055, China

Keywords: gear ring, lifting, finite element analysis, deformation.

\begin{abstract}
Gear is the key part of cement rotary kiln, and lifting the process of deformation delivers a direct impact upon gear ring manufacturing precision. This paper takes the 10630. 249mm gear ring of rotary kiln as an example, expounds the system of lifting method for gear ring and selecting the optimization lifting point. After gear ring model is established using PRO/E, and simulation and calculation the deformation of gear ring on lifting, so as to determine the optimal solution and the best lifting points. The result not only reduce the deformation to ensure accuracy of the gear ring manufacturing, but also provide a reference work for gear ring lifting in engineering practice.
\end{abstract}

\section{Introduction}

Ring gear is mainly used in the cement industry kiln and edge drive tube mill drive, belonging to the core equipment in cement components. With the expansion of the cement production scale, equipment specification has also increased, oversize gear ring diameter and weight increases, the strength and rigidity of the gear ring is reduced, so to control the deformation of the gear ring to become the focus of manufacturing. Increasing the gear ring of the weight and size leads to a reduction of strength and rigidity, more difficult to bring to the lifting work. The relationship between the deformation of the ring gear to the manufacturing precision of gear ring have been changed, whether through acceptance testing, whether onsite installation tolerances, and ultimately affect the smooth operation. Therefore the development of rational lifting program, select the best lifting point decrease in lifting ring deformation process manufacturing problems became manufacturers and installers of great concern.

Despite the lifting of the kiln ring deformation is very concerned, but based on experience at home and abroad generally inferred to determine the lifting scheme. For a more detailed understanding of deformation and quantitative analysis of the gear ring when lifting the deformation, this paper will conduct the actual structure for the $\Phi 10630.249 \mathrm{~mm}$ of the simulation, the finite element analysis on the different lifting deformation mode of gear ring, finally discusses the optimal gear ring lifting scheme.

\section{Type of gear ring and lifting method}

\section{Make the scheme lifting gear ring}

The rotary kiln ring gear 61.888 tons of weight, diameter of $\Phi 10630.249 \mathrm{~mm}$ and the material for the 40CrNi2Mo alloy structural steel, which belongs to the oversize gear ring. Since the dimensions and mass of the ring gear of relatively large, in the production and manufacturing process, in order to facilitate transportation and installation site of the way, the common approach is to divide it into two half ring production factory or divided into four 1/4 ring production factory, the installation of the ring and then a half or $1 / 4$ of the ring together. 


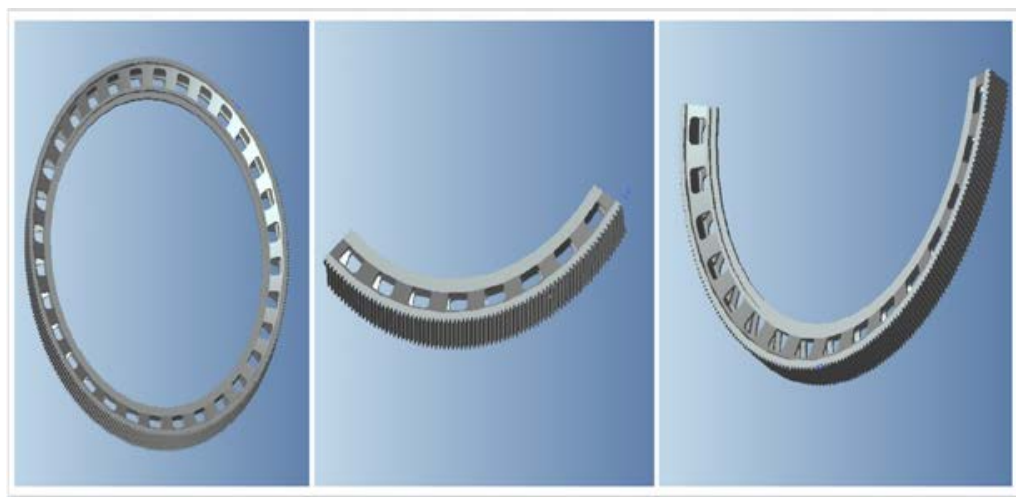

Fig.1: Three type of ring gear

(Whole ring gear;1/4 ring gear; semiring gear)

The most common oversize ring gear of lifting have three kinds: horizontal lifting, flip lifting, vertical lifting.

For horizontal lifting by lightening hole on the ring gear. Respectively, using a pair of wire rope through the ring gear symmetrical position weight lifting holes for modular full circle ring, lifting points should be distributed in a combination of both sides, when the ring also can be used on vertical lathes way that lifting.
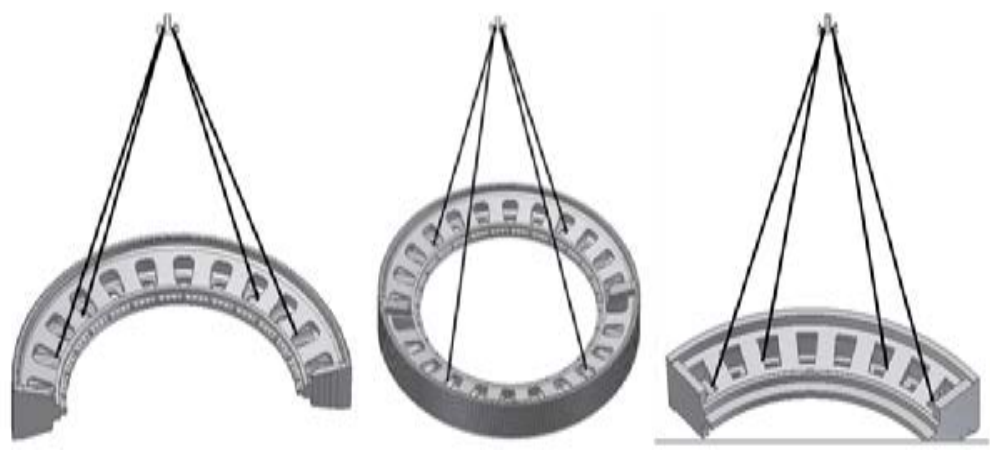

Fig.2: Horizontal lifting scheme

(semi_ring horizontal lifting; whole ring horizontal lifting;1/4 ring horizontal lifting)

Vertical lifting by lightening hole on the ring gear. Using a pair of wire rope through the ring gear symmetric four lightening holes suitable, making the semi_ring, 1/4ring gear combined with face lifting on.
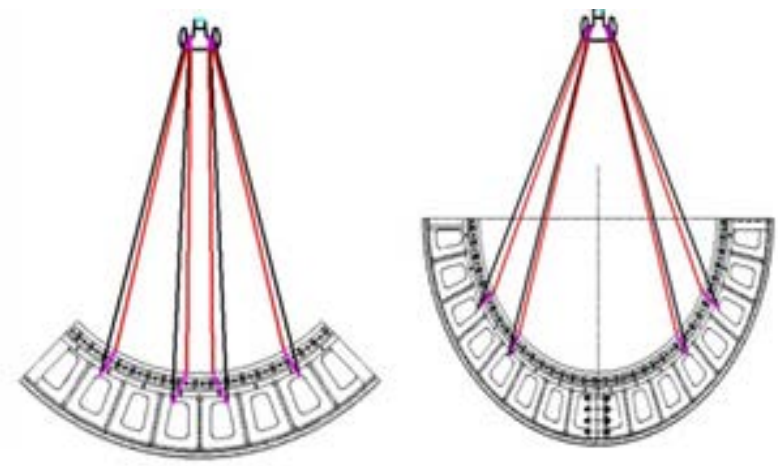

Fig.3: Vertical lifting scheme

(1/4 ring vertical lifting; semiring vertical lifting)

For flip by lightening hole on the ring gear. Respectively, using a pair of wire rope through four holes suitable lightening holes oversize lifting gear ring. For modular full circle ring, lifting points should be distributed on both sides of the joint surface. Two inner wire rope lifting points should be 
evenly distributed in the combination of both side surfaces, the inner side of both outer rope hoisting rope lifting points should pitch interval 1, 2 lightening holes, and symmetrical distribution.

In order to systematic and comprehensive on the ring gear lifting deformation analysis, it is combined with the actual structure of the ring, select erection of the three most commonly used lifting methods, horizontal lifting, vertical lifting and flip lifting. Make the scheme lifting gear ring as follows, whole ring horizontal lifting, whole ring flip, semiring horizontal lifting, semiring flip, semiring vertical lifting,1/4 ring horizontal lifting, flip and vertical lifting.
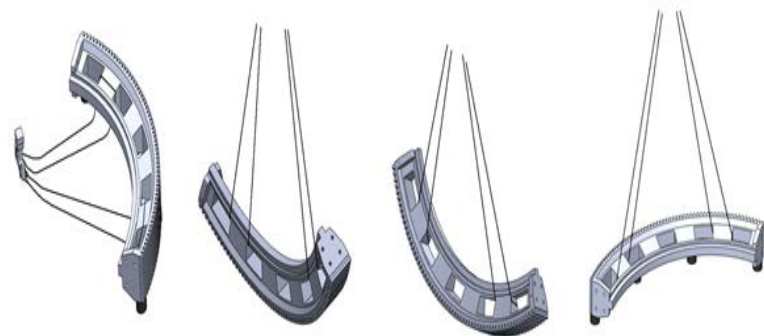

Fig.4: Flip lifting scheme

(1/4 ring flip lifting)

Table.1: The lifting scheme of gear ring

\begin{tabular}{|c|c|c|c|}
\hline Number & Scheme 1 & Scheme 2 & Scheme 3 \\
\hline Whole ring & Horizontal lifting & & Flip lifting \\
\hline Semi_ring & Horizontal lifting & Vertical lifting & Flip lifting \\
\hline 1/4ring & Horizontal lifting & Vertical lifting & Flip lifting \\
\hline
\end{tabular}

\section{The analysis of lifting point setting for gear ring}

When lifting objects, must be make sure that the center of gravity of the balance. Oversize ring gear belongs to the symmetric structure, the setting of lifting points should also be symmetrical distribution. Because of this paper the lifting method is lightening whole wire rope through the ring gear for lifting, so are circumferential arranged holes on the ring gear are lifting points. For the same ring, with a lifting method, the choice of different lifting points, the impact on the accuracy of the deformation and the ring gear is different.

\section{Deformation analysis of oversize ring gear lifting}

\section{The finite element method and its implementation process}

The finite element analysis method is to use mathematical approximation to simulate the real physical system. Also use simple and interacting elements, with a limited number of unknowns to approximate the real system with infinite unknown quantity.

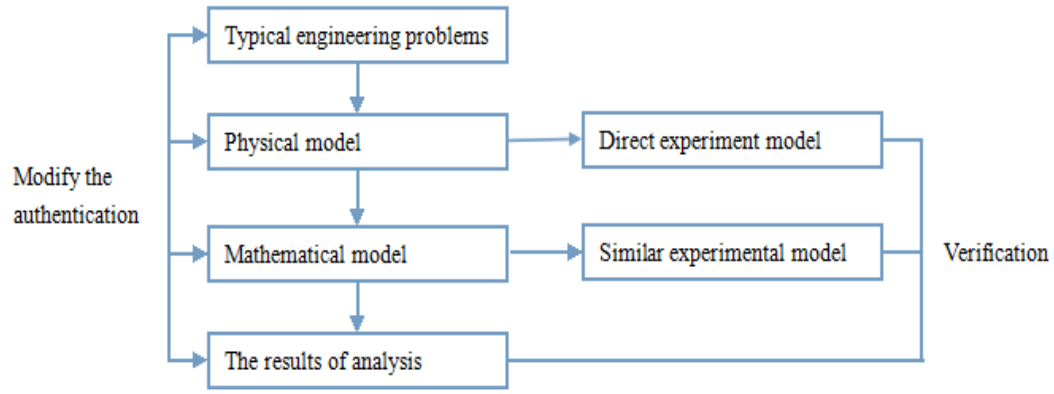

Fig.5: Modeling and analysis of process engineering problems

Finite element method has been widely used in various engineering fields, such In engineering practice, finite element analysis method usually has three main parts. Model establishment stage. 
To establish the required object model analysis in finite element software, the simulation model, the geometry of the part is divided into a plurality of discrete units, units are connected by discrete points. The whole process takes work includes: analysis of structural form, to establish the structural model, meshing, the definition of element properties, inspection unit quality and defining, the boundary condition and the applied load etc. This will produce three types of data, respectively, and the boundary conditions of node unit.

The solution phase.

After the calculation of the completion stage is modeling, the pretreatment model input for numerical calculation of finite element program, and output the corresponding results. The main work includes: the formation of the overall matrix, the treatment of boundary conditions and solution of the characteristic equation.

The post processing stage.

The solution is completed, need to make corresponding processing to the calculation results, and it will be in a corresponding manner. Easy to analyze and evaluate the analysis model, and proposes the corresponding optimization or improvement suggestions.

In the three stage, the pretreatment stage is the key of the whole analysis process, it bears the responsibility for providing the data calculation, accuracy and rationality of the model all the calculated results are closely related. At the same time complexity condition complexity and gear ring structure increased the difficulty of establishing model, the relevant technical personnel requirements also increase. Preprocessing stage is the core of the whole process of analysis, but also with the longest part, so to improve the quality and efficiency analysis must do a thorough research on this process.

\section{Oversize gear ring lifting steps and setting of lifting points}

Lifting method is the core of the ring gear lifting process, select the appropriate lifting method can improve the lifting efficiency, ensure the smooth process of lifting. The selection of lifting method should follow the safety, fast, economy, the principle of orderly. Like lifting oversize gear ring, with great risk, once the accident happens, it may lead to significant property damage and personal injury. And the safety of the lifting process and whether or not the key lies in the selection of lifting method.

Select lifting scientific method, should follow these steps.

i) Technical feasibility

There are technical limitations of each lifting method are inevitable, every lifting construction are different. Technical feasibility demonstration, is according to the characteristics of the equipment and site conditions, research is feasible in the technology of the lifting method.

ii)Safety analysis

The lifting ring gear must be combined with the specific situation, to the feasible method of each kind of technology from technology analysis and comparison. Find the unsafe factors and solving method, reliability and careful analysis of these methods. Safety analysis includes a ring gear, the deformation and failure in the process of lifting, and personnel safety.

iii)Cost analysis

Complete the project with low cost, to earn a reasonable profit, is the construction of the objective. Therefore, must accord with the requirements of lifting scheme of security and progress of the lowest cost accounting. Selection of lifting method which is low cost, but the firm is not allowed in order to reduce the cost and the selection of lifting method of security is not good.

Lifting point as the connection point between the gear ring and a lifting rope operation, select the appropriate lifting point is a very important point in the lifting plan. Lifting point selection is not appropriate, it will make the rope uneven stress, resulting in rotation of the ring gear or overturn. Setting of lifting points according to the capacity of gear ring structure of the weight and center of gravity and lifting equipment to determine. 


\section{To establish the finite element model of the gear ring}

The application of finite element software, large gear ring in the lifting process, bearing load for structure weight load. In the self-weight load, the internal structure will produce stress and displacement. Stress exceeds the allowable value or deformed serious will cause the structure damage and deformation of the ring gear. So, in the lifting process must ensure that the structure has enough stiffness and strength. Thus, for the finite element model of the gear ring is necessary.

In the $\Phi 10630.249 \mathrm{~mm}$ ring gear as the research object, will use three ring gear model in PRO/E software to establish the exchange using finite element analysis software ANSYS through data. Since the paper deformation in the entire ring gear, in order to simplify the model and therefore does not affect the results, when the geometric model, ignoring the structure of the overall stiffness and strength of the local fine structure has little influence, such as the ring gear of the chamfer, rounded, small holes, teeth etc..., in order to improve the efficiency and computing speed of the finite element model. Secondly, when the ring gear meshing, mesh trial results, until the grid convergence, thus meshing complete[3].Ring gear using three dimensional solid elements, material properties as shown in Table 2.The ring gear finite element mesh model is built as shown in Figure 4.

Table 2 Material properties of gear ring

\begin{tabular}{|c|c|c|c|}
\hline Material & Elastic Modulus/Gpa & Poisson’s ratio & Density $\left(\mathrm{kg}_{\mathrm{m}} \mathrm{m}^{-3}\right)$ \\
\hline 40CrNi2Mo & 206 & 0.3 & 7850 \\
\hline
\end{tabular}

\section{The boundary conditions and the solution of load}

In the process of lifting, the tension load gear ring subject to gravity and the steel wire rope. Due to the deformation of wire rope on the impact of overall deformation of the ring gear is very limited. Therefore, constraints imposed on the wire rope, wire rope to the ring gear influence would not have considered, mainly according to the different lifting method, freedom and reasonable constraint lifting points in the ring gear radial and axial. While the ring gear of its own weight is achieved by applying the acceleration of gravity.

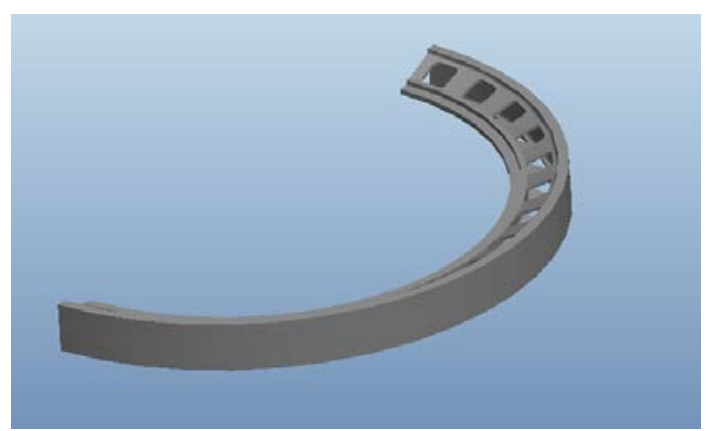

Fig.6: The actual model after treatment

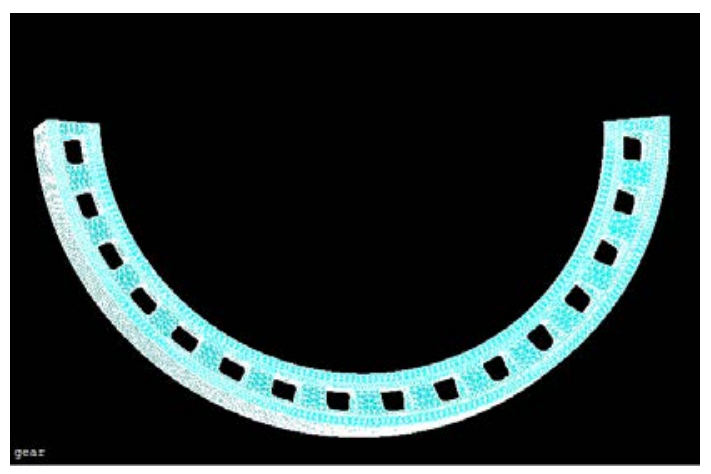

Fig.7: Semi_ring gear meshing 


\section{Conclusion}

Ring gear horizontal lifting and vertical lifting deformation problems using static deformation analysis, the flip with spectrum analysis .Not only optimize lifting scheme, but also determine the optimum points.

\section{References}

[1] Guoqiang Wang: Numerical Simulation Technology of Practical Engineering and its Practice on ANSYS (Northwestern Polytechnical University Press, Xi'an 1999), (In Chinese)

[2] LuoYang Mining Machinery Engineering Design (Special lifting ring gear specifications. (In Chinese)

[3] Min Song, Chengshan Li and Yuxin Duan:Drum mixer lifting cylinder deformation analysis, Nonferrous Mining and Metallurgy Vol. 6 (2012) (In Chinese)

[4]Jingwei Xing: Large machinery and equipment and materials to explore lifting safety, Water Science and Technology and Economy Vol. 17 (2011) No. 8(In Chinese).

[5] Kyungsik choi.Lifting Analysis for Heavy Ship_hull Blocks Using 4 Cranes[J].International Journal of Offshore and Polar Engineering, 2005, 15(1):62-64.

[6] Gang Xiong, Research on integral assembly lifting of large and Medium-sized bulk carrier's superstructure [D].May, 2013. 